\title{
Time discreteness \& Wave function aliasing
}

\section{Alexander Zaslavsky}

National Mining University, Ukraine and Web-Research Institute of the nature of time based Russian interdisciplinary seminar Temporology working at Moscow State University.

Keywords: state, stream of events, time quantum, aliasing, wave function, energy.

\begin{abstract}
The conditions of arising and some effects of physical phenomenon that may be found in the process of observing high energy particles are considered in this work. This phenomenon is consequence of time discreteness. When the repetition frequency of a particle discrete state proves to be commensurable with its own frequency (energy), the aliasing effect of its wave function may take place. The wave function aliasing limits the particle energy spectrum giving rise to the uninvestigated abnormal modes of movement. In view of the fact the discreteness of particle states at a time is a prerequisite for aliasing, the effect under consideration, in case of its experimental verification, may serve a proof of this discreteness.
\end{abstract}

\section{Introduction}

The ideas of space and time discreteness, as well as the problems of existence of a fundamental length and a fundamental time $[1,2,3]$ are becoming more and more popular subjects of discussions in recent years. However, a question being raised as to whether the time is continuous or discrete can't be finally solved on the basis of mere theoretical arguments. There is a need for certain proof- of-concept experiment that should cross the T's. A physical phenomenon that may serve a base for such experiment is investigated and forecasted in this work on the grounds of hypothesis as to the discreteness of time. The states of unbound particle discretely (intermittently) moving in space are construed as a stream of events divided by random time intervals different from zero. These intervals characterizing the repetition frequency of particle states due to certain objective relativistic laws on the average prove to be functionally related to its movement speed. Upon gaining the speed wherein the average repetition frequency of particle instantaneous states becomes less than a double frequency of its wave function, the aliasing effect occurs according to the well known Kotelnikov - Shannon sampling theorem. It is worth saying, that the aliasing effect is well known in the application tasks connected with the digital signal processing. Nevertheless it wasn't studied asregards fundamental physical problems. It may be assumed that this has happened at least for two reasons. First, nothing of this kind has been observed at present with the energy levels attainable on the basis of experiments conducted with elementary particles; secondly, the Time Continuity Concept dominating in physics excludes this phenomenon as a natural one. However, the energy levels attainable on the basis of experiments conducted with elementary particles increase, and if the level ensuring identification of wave function aliasing is attained, this will be a sign of the need for revision of the Time and Movement Concept.

\section{Principle of Relativity for the streams of events}

A random succession of instantaneous events is identified as a stream in the theory of random processes. We shall consider the instantaneous states of elementary particle representing an object under observation as these events. The concept "state of particle" may convey different meaning depending on a perspective from which this object is considered. The state may be characterized by the coordinates of a point in space (phase, Hilbert, Minkowsky, etc.), speed, energy, impulse and other dynamic parameters. We designate as an event any parameter set determining particle instantaneous state supplemented with its occurrence time. Space-time point in the relativity theory is a common example of the event. A certain set of the events of this nature 
represents the fact referred to as a particle. The elementary character of a particle as a stream of events means that it is considered as an integer i.e. a system wherein a single state strictly corresponds to the instant of time. We shall consider the state of elementary particle in the stream of events as a certain generalized characteristic of the instant of time. The assumption that continuous time possesses infinitely large time density along with a certain a priori property being characterized as duration is inherent in the conventional approaches. The states arranging between the events of particle in the chain of its own events will be always found in the continuous time for any two states of a particle. Therefore, there are no any related events (i.e. the events following directly one after another) in the continuous time. This work studies the alternate (event-driven) concept according to which the time doesn't possess a priori duration being measured by the final number of events. Suppose there is a certain elementary particle relative to which the movements of other observable particles are being studied. Instantaneous states of this particle correspond to the instants of its own time in a one-to- one manner. The stream of its states is a referent criterion of its own time. Therefore, in the frame of reference connected with a particle a discrete time is measured by the number of its states. If an observer transforms a certain chain of events into the chain being the time for him, this observer indentifies the observable sequence of events as a stream of events of observed object. The time interval between any two events in this stream is measured by a number of the instants of time (states of frame of reference) contained between them.

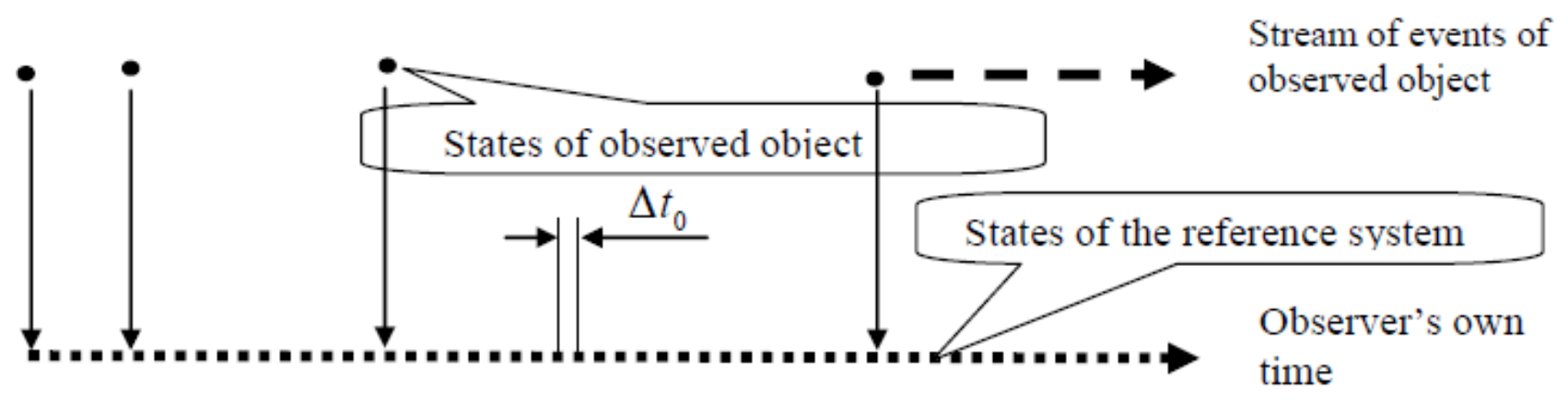

Fig. 1. Picture of event-driven time concept

Meanwhile, the observer's time is identified with own time of the particle aforementioned, thus, constant - own time quantum $\Delta t_{0}$ is put in correspondence with a pair of related events (instants). Its duration is principally unmeasured in own time, on the contrary, the observer's own time is measured by a number of events - instants, i.e. the number of time quanta. Suppose there is the second observer. As with the first case, the observer's own time is measured by a number of elementary events being identified to the instants of time. Similarly to the first one, this observer puts a constant - own time quantum in correspondence with a random pair of own related events (instants). By virtue of the aforementioned considerations, this constant is also immeasurable in the second observer's own time because own time is measured by the number of quanta. In other words, the first observer's time quantum, as a matter of principle, may be measured in the second observer's time, but not in the own time. For example, I can measure averaged duration of elementary interval (one second) with the help of my watch in the watch of my interlocutor. This interlocutor can do the same as regards my watch by means of his one, but I can't measure the duration of one second in my watch by the time shown by it in any way. Therefore, the own time quantum accurate to within measurement units is a universal constant just owing to impossibility to measure it in the own time. Each observer surely can assume the own time value at random, but this fact will only complicate the calculation in which the time is present without any changes per se due to the need to take into consideration the measurement unit ratio. For example, suppose the own time quantum of one observer is equal to a definite value with the own time quantum of another observer being equal to other value. So long as both quanta are the constants, their ratio is a constant too. Therefore, it may be assumed that the quanta are equal but, the measurement units are 
related by different observers as a constant. Meanwhile, the time is measured by the number of quanta in the observer's time frame.

$$
\begin{aligned}
& \forall \Delta t\left(0<\Delta t_{0} \leq \Delta t, \Delta t_{0}=\text { const }\right), \\
& \Delta t=\Delta t_{0} \tau
\end{aligned}
$$

The streams in which the events appear one by one so that a probability to find two or more events in the short time interval tends toward zero are known as the ordinary streams. The ordinariness of a stream indicates that simultaneous events are absent in it. For example, suppose that an object under observation is represented by a sequence of states of a certain system in the aggregate. So long as the system is only in any single state in every instant of time, the stream of its states is an ordinary one. Let us agree to mark the values relating to an object under observation by index $k$ and the values relating to the observer leave without any index. Let us write $\tau_{k}(t, \Delta t)$ for the number of events having happened within $(t, t+\Delta t)$ time interval. It is supposed here and elsewhere that an observer presenting reality in an unbiased manner is recording every state of an object under observation. The distribution series of $\tau_{k}(t, \Delta t)$ random variable are as follows:

$$
\tau_{k}(t, \Delta t):\left|\frac{0}{p_{0}^{k}(t, \Delta t)}\right|\left|\frac{1}{p_{1}^{k}(t, \Delta t)}\right||\cdots|,
$$

whereon the possible values of numbers of events $2,3, \ldots$, are shown at the top of the column with elision marks with the probabilities corresponding to them being shown at the bottom.

The following normalization condition is satisfied for any $(t, \Delta t)$ pair

$$
p_{0}^{k}(t, \Delta t)+p_{1}^{k}(t, \Delta t)+p_{2}^{k}(t, \Delta t)+\ldots=1
$$

The mathematical expectation of $\tau_{k}(t, \Delta t)$, random value is equal to the following total:

$$
M\left[\tau_{k}(t, \Delta t)\right]=0 \cdot p_{0}^{k}(t, \Delta t)+1 \cdot p_{1}^{k}(t, \Delta t)+2 \cdot p_{2}^{k}(t, \Delta t)+\ldots .
$$

In case of ordinary stream with $\Delta \mathrm{t} \rightarrow 0$ the probability for appearance of two or more events in $\Delta \mathrm{t}$ interval tends towards zero. Therefore, the mathematical expectation of $\tau_{k}(t, \Delta t)$, random value in the limit tends to the value as follows:

$$
\mu\left[\tau_{k}(t, \Delta t)\right]=\lim _{\Delta t \rightarrow 0} M\left[\tau_{k}(t, \Delta t)\right]=p_{1}^{k}(t, \Delta t)
$$

Acting on the basis of the principle of relativity for the streams of events, we shall determine own time of an object under observation from (1) having substituted $\mu\left[\tau_{k}(t, \Delta t)\right]$ mathematical expectation of the number of events of an object under observation within $(t, t+\Delta t)$ time interval for a number of events $\tau$ taken by an observer as the instants of own time. $\Delta t_{k}=\Delta t_{0} \mu\left[\tau_{k}(t, \Delta t)\right]$. Let us determine $\Delta t_{k} / \Delta t$ own time of an object under observation-to-observer's own time ratio for an ordinary stream.

$$
\frac{\Delta t_{k}}{\Delta t}=\Delta t_{0} \frac{\mu\left[\tau_{k}(t, \Delta t)\right]}{\Delta t} .
$$


Value $\sigma(t)=\frac{\mu\left[\tau_{k}(t, \Delta t)\right]}{\Delta t}$ is known as the average density (intensity) of ordinary stream of events in the theory of random processes. It represents the mathematical expectation of a number of events falling on the unit of time in segment $\Delta t$ adjacent to the current instant of time t. Therefore, the expression (7) may be presented in the following form:

$$
\frac{\Delta t_{k}}{\Delta t}=\Delta t_{0} \sigma(t)
$$

Let us evaluate own time intervals of an object under observation-to-observer's own time intervals ratio. To do so, let us make use of $\Delta t_{0}$ time interval determination as the least possible one between two adjoint states of any system. In view of the fact that value $\Delta t_{0}$ in (7) according to determination (1) is less than any value given in the denominator and with due regard for (6) we shall derive $\frac{\Delta t_{k}}{\Delta t} \leq p_{1}^{k}(t, \Delta t) \leq 1$. This inequality means that own time of an object under observation isn't passing more quickly than the observer's time. If an observer finds instantaneous state of an object under observation with the probability 1 in every $\Delta t_{0}$ time interval, this means that their time is passing equally. If $p_{1}^{k}(t, \Delta t)<1$; the time of an object under observation is passing slower than the observer's time.

From the stationary observer's point of view the adjoint states of moving particle are divided in the stream of events by random time intervals $\Delta t_{1}, \Delta t_{2}, \ldots, \Delta t_{i}, \ldots$ These time intervals are measured by the number of instantaneous states of a particle stationary relative to the observer. The discrete space positions of a particle correspond to the discrete instants of time. And at the same time, the particle being at the instant of time $t_{0}$ in the point $R_{0}$ passes intermediate states and at the instant of time $\left(t_{0}+\Delta t_{i}\right)$ finds itself in the point $\left(\mathbf{R}_{0}+\Delta \mathbf{R}_{i}\right)$, herein $\Delta \mathbf{R}_{i}$ designates random increments of radius vector indicating the space position of a particle. When a particle is regarded as a discrete stream of events, we must assume that this particle actually exists only at the fixed random instants of time and only at the fixed random points in space. The concept of particle speed of movement in space is non-applicable to the process of single-shot change of its state

$$
\text { However, one may speak about the average speed } \overline{\mathbf{V}}=\frac{\sum_{i=1}^{N} \Delta \mathbf{R}_{i}}{\sum_{i=1}^{N} \Delta t_{i}}
$$

multiple changes of its state. The less time interval $\Delta \bar{t}$ is in relation to the time interval $\Delta T$ 'on which the process of movement is examined, the more precisely this value corresponds to the ordinary concept of speed. In other words, the particle speed of movement represents a limit to which the average drift speed of its state $V=\lim _{\Delta t / \Delta T \rightarrow 0} \overline{\mathbf{V}}$ is converging.

Suppose a particle moving at a speed of $\mathbf{V}$ relative to the inertial reference system represents an object under observation. If this is the case, own time of an object under observation-to-observer's own time ratio, as is well known, is equal to:

$$
\frac{\Delta t_{k}}{\Delta t}=\sqrt{1-\frac{\mathbf{V}^{2}}{c^{2}}} \leq 1 .
$$

The special relativity theory establishes a functional connection (9) between the time intervals and speed of movement. In consideration of time filling with the events, the principle of relativity for the streams of events establishes a functional connection (8) between the time intervals and the 
density. Having compared (8) and (9), we shall derive a formula connecting the density of a stream of events of the particle with its speed of movement in the inertial reference system.

$$
\sigma(\mathbf{V})=\frac{1}{\Delta t_{0}} \sqrt{1-\frac{\mathbf{V}^{2}}{c^{2}}}
$$

This formula implies that the average number of events of a moving particle falling on the unit of time is reducing with increase of its speed. When the particle under observation is immovable in respect to an observer, the density of its stream of events is equal to the density of observer's time. This means that an observer is snapping instantaneous state of the particle under observation in every elementary time interval. With increase of the speed of movement of a particle its instantaneous states fail getting in every elementary time interval of an observer. Having attained the limit speed of movement, the instantaneous states of particle don't get in any elementary time interval of an observer. Actually, maybe this is the phenomenon explaining the fact that the speed of light is unattainable for the particles being observed in space as the objects in movement.

\section{Substitute (aliasing) of Particle Frequency}

The state of moving particle is described in quantum mechanics by a wave function as follows:

$$
\psi(\mathbf{r}, t)=C e^{j(\omega t-\mathbf{k r})}=A(\mathbf{r}) e^{j(\omega t)},
$$

Whereon $\omega=2 \pi \nu^{\prime}$ wave frequency, $\mathbf{r}$ is radius vector of random point in space, $\mathbf{t}$ is time, $\mathbf{k}$ is wave vector. The frequency and wave vector are connected with the energy and momentum of a particle by well known de Broglie relations $E=\hbar \omega, \mathbf{p}=\hbar \mathbf{k}$, whereon $\hbar$ (reduced) Planckian constant. The conventional concept determines a wave function simultaneously in all points of continuous space and in any moment of continuous time

However, the approaches substantiating time-space discreteness are well known at present. When a particle is regarded as a discrete stream of events, it is necessary to interpret its wave function in an appropriate manner. The results obtained by Shan Gao [4] are interesting from this point of view. Analyzing the results of interpretation of wave function of the quantum system, he obtains the following: the wave function should be regarded not as a field, but as a stream of particle states in discrete time-space. It is represented by the aggregate of sampled values $\psi\left(\mathbf{r}_{\tau}, \tau\right)$ corresponding to the discrete instants of time $(0,1,2, \ldots \tau, \ldots)$

$$
\psi\left(\mathbf{r}_{\tau}, \tau\right)=\int_{-\infty}^{\infty} \psi(\mathbf{r}, t) \delta(\tau \Delta \bar{t}-t) d t
$$

whereon $\psi(\mathbf{r}, t)$ is the wave function in which a particle in the continuous time-space is described. This function may be recovered according to sampled values on the basis of Kotelnikov - Shannon sampling theorem. Analyzing a particle in the discrete time-space in interaction with its environment, we have to perform a procedure for restoration of continuity in connection with integro-differential nature of the laws applied to describe this interaction.

The condition for reasonable restoration of the wave function continuity in time according to sampling theorem is of the form as follows:

$$
\frac{\omega}{2 \pi} \leq F=\frac{\sigma(\mathbf{V})}{2} \text {, }
$$


whereon $\mathrm{F}$ is the Nyquist frequency. If the time is considered to be continuous $(0 \Delta t=0)$, the density of a stream of particles according to (10) is infinitely large. And at the same time the inequality (12) is performed for any frequency of the wave function. If the time is discrete ( $0 \Delta \mathrm{t}>$ 0 ), namely being a basis for the event concept, then (11) is adequately describing the wave function only with the frequencies being not higher than the Nyquist frequency.

Substituting value $\sigma$ from (10), we shall obtain the inequality as follows:

$$
\frac{1}{\Delta t_{0}} \sqrt{1-\frac{\mathbf{V}^{2}}{c^{2}}} \geq \frac{\omega}{\pi},
$$

whence it follows that

$$
\frac{|\mathbf{V}|}{c} \leq \sqrt{1-\frac{\omega^{2}}{\pi^{2}} \Delta t_{0}^{2}} .
$$

The result obtained may be interpreted as stated below. So far as the speed of a particle doesn't exceed the value:

$$
c_{F}=c \sqrt{1-\frac{\omega^{2}}{\pi^{2}} \Delta t_{0}^{2}},
$$

the wave function characterized by frequencymay be unambiguously restored by $\omega$ a discrete sequence of its instantaneous values following one after another with the intensity

$$
\sigma=\frac{1}{\Delta t_{0}} \sqrt{1-\frac{V^{2}}{c^{2}}} .
$$

Let us see what will happen if a particle exceeds speed $c_{F}$. Having used the method cited by R. Otnes and L. Enochson [5], let us express the time of observation $t$ through the average period of instantaneous states of a particle in the stream of events $t=\frac{\tau}{\sigma}, \tau=0,1,2, \ldots$

$$
\psi=A(\mathbf{r}) e^{j \frac{\omega}{\sigma} \tau}
$$

we shall also present ratio $\frac{\omega}{\sigma}$ in the form of integral $z$ and fractional $q$ parts of $\pi$ character

$$
\frac{\omega}{\sigma}=z \pi+q \pi
$$

as this takes place, the wave function will be transformed to the form as follows:

$$
\psi=A(\mathbf{r}) e^{j z \pi \tau} e^{j q \pi \tau}
$$

Now we have two opportunities depending upon parity and imparity of value $Z$

1) If $\boldsymbol{z}$ is even number, ${ }^{e^{j z \pi \tau}}=1$ for all $\tau$ in correspondence with the rotation of vector $\psi$ in the plane of complex numbers to the angle multiple of $2 \pi$. 
2) If $\boldsymbol{z}$ is odd number, ${ }^{e^{j z \pi \tau}}=(-1)^{\tau}$ for all $\tau$ in correspondence with the rotation of vector $\psi$ in the plane of complex numbers to the angle multiple of $\pi$.

\[ \frac{\tau}{\sigma}=t \]
Taking into account that, let us rewrite angle q $\pi \tau$
$q \pi \tau=(\omega-z \pi \sigma) \frac{\tau}{\sigma}=\omega^{\prime \prime} t, \quad \mathrm{z}=0,2,4, \ldots$,

whence it's seen that $\omega=2 \pi \nu$ is substituted for the frequency:

$$
\omega^{\prime \prime}=\omega-\pi \sigma z=\omega-\frac{\pi}{\Delta t_{0}} z \sqrt{1-\frac{V^{2}}{c^{2}}}, \quad \mathrm{Z}=0,2,4, \ldots
$$

In the second example let us take into account the following: $(-1)^{\tau}=\cos (\pi \tau), \sin (\pi \tau) \equiv 0$ Let us present complex number $e^{j z \pi t} e^{j q \pi \tau}$ in the trigonometric representation having supplemented it with intentionally zero summands:

$$
\begin{aligned}
& e^{j z \pi \tau} e^{j q \pi \tau}=\cos (\pi \tau) \cos (\pi q \tau)+j \cos (\pi \tau) \sin (\pi q \tau)+ \\
& +\sin (\pi \tau) \sin (\pi q \tau)-j \sin (\pi \tau) \cos (\pi q \tau)= \\
& =\cos (\pi \tau(1-q))-j \sin (\pi \tau(1-q)) .
\end{aligned}
$$

Therefore, for odd numbers $\boldsymbol{z}$ we have:

$$
e^{j z \pi \tau} e^{j q \pi \tau}=e^{-j \pi \tau(1-q)} \text {. }
$$

Let us rewrite angle $\pi \tau(1-\mathrm{q})$ in the following form:

$\pi \tau(1-q)=(\pi \sigma-(\omega-z \pi \sigma)) \frac{\tau}{\sigma}=\left(2 \pi F-\omega^{\prime \prime}\right) t, \quad \mathrm{z}=1,3, \ldots$

In this case the wave frequency is substituted for the following frequency:

$$
\omega^{\prime}=2 \pi F-\omega^{\prime \prime}=2 \pi F-\omega+\frac{\pi}{\Delta t_{0}} z \sqrt{1-\frac{V^{2}}{c^{2}}},
$$

if $z=1$, we shall obtain:

$$
\omega^{\prime}=\frac{2 \pi}{\Delta t_{0}} \sqrt{1-\frac{V^{2}}{c^{2}}}-\omega
$$

Thus it's seen, that if the density of stream of events of a particle under observation is being reduced due to increase of its speed up to critical value, $\sigma=2 F=\frac{\omega}{\pi}$ , the frequency (energy) is 
being substituted. The frequency line shown in the diagram of frequency dependence on speed (Fig. 2) doesn't monotonically go into infinity, but "is folded". This has stipulated the conventional designation of the Nyquist frequency by letter F (from English Fold). Due to this phenomenon the frequencies differing from the ones that may be observed if the density of a stream of events would be infinitely large may appear the frequency spectrum under observation. Let us consider a dependence of frequency (energy) of an unbound particle on the speed of movement with the account taken of limited density of its instantaneous state stream.

The energy and frequency of an unbound particle with mass $m$ in the inertial reference system, as is well known, are equal to: $E=\frac{m c^{2}}{\sqrt{1-\frac{V^{2}}{c^{2}}}}, \omega=\frac{m c^{2}}{\hbar \sqrt{1-\frac{V^{2}}{c^{2}}}}$ attains value $c_{F}$. Further increase of the speed will lead to the frequency substitution according to (15).

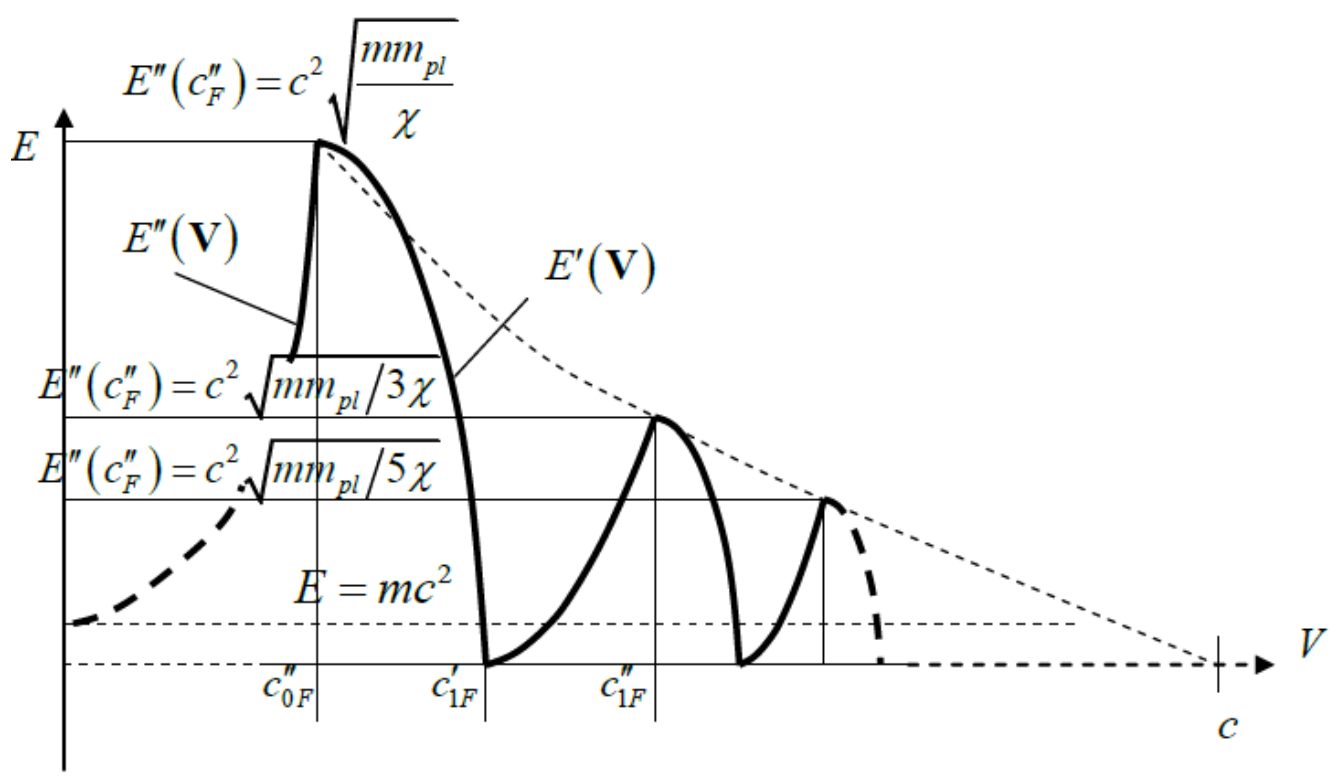

Fig. 2. Specificities of energy spectrum of ultrarelativistic particle due to frequency substitution

$$
\begin{aligned}
& \frac{m c^{2}}{\hbar \sqrt{1-\left(\frac{c_{F}}{c}\right)^{2}}} \text { and substitution frequency } \\
& \text { Reference frequency } \\
& \text { speed }|\mathbf{V}|=c_{F} \text { equal to: } \\
& \frac{m c^{2}}{\hbar \sqrt{1-\left(\frac{c_{F}}{c}\right)^{2}}}=\frac{\pi}{\Delta t_{0}} \sqrt{1-\left(\frac{c_{F}}{c}\right)^{2}} .
\end{aligned}
$$


From this equation we shall find:

$$
\frac{c_{F}}{c}=\sqrt{1-\frac{m c^{2}}{\pi \hbar} \Delta t_{0}}
$$

Let us express ratio $\frac{m c^{2}}{\pi \hbar}$ through Planckian time $t_{P l}=\sqrt{\frac{\hbar G}{c^{5}}}$ and Planckian mass $m_{P l}=\sqrt{\frac{\hbar c}{G}}$. Having multiplied these two values we shall obtain: $\frac{c^{2}}{\hbar}=\frac{1}{t_{P l} m_{P l}}$, whence $\frac{m c^{2}}{\pi \hbar}=\frac{m}{m_{P l}} \frac{\Delta t_{0}}{\pi t_{P l}}$ follows.

Let us denote dimensionless constant

$$
\chi=\frac{\Delta t_{0}}{\pi t_{P l}}
$$

indicating elementary reduced time interval $\frac{\Delta t_{0}}{\pi}$ to Planckian time ratio. $t_{P l}$ In view of (25) the expression (24) is simplified for the critical speed of a particle wherein the aliasing arises

$$
\frac{c_{F}}{c}=\sqrt{1-\chi \frac{m}{m_{P l}}}
$$

If the speed of a particle increases over value $c_{F}$, its frequency (energy) is reduced from the stationary observer's point of view due to frequency substitution as may be inferred from (21) contrary to expected increase.

Using continuous numbering $z=0,1,2,3, \ldots$ we shall obtain the following value of substituted frequency for even $z^{\prime \prime}=2 z$ folds:

$$
\omega^{\prime \prime}=\omega-\frac{\pi}{\Delta t_{0}} 2 z \sqrt{1-\frac{V^{2}}{c^{2}}}, \quad \mathrm{Z}=0,1,2,3, \ldots
$$

The value of substituted frequency for odd $z^{\prime}=2 z \pm 1_{\text {values is equal to: }}$

$$
\omega^{\prime}=2 \pi F-\omega+\frac{\pi}{\Delta t_{0}}(2 z \pm 1) \sqrt{1-\frac{V^{2}}{c^{2}}}, \quad \mathrm{z}=0,1,2,3, \ldots
$$

Let us determine the speed of a particle movement at the conjunction points of the folds. Let us write $c_{z F}^{\prime \prime}$ for the critical speed in the transition point of even fold into the odd one and $c_{z F}^{\prime}$ for the critical speed in the transition point of odd fold into the even one. 
The equality $\omega^{\prime \prime}\left(c_{z F}^{\prime \prime}\right)=\omega^{\prime}\left(c_{z F}^{\prime \prime}\right)$ is a condition of conjunction when transition from the even fold $z^{\prime \prime}=0,2,4, \ldots$ to the odd fold $z^{\prime}=1,3,5, \ldots$ The equality $\omega^{\prime}\left(c_{z F}^{\prime}\right)=\omega^{\prime \prime}\left(c_{z F}^{\prime}\right)$ is

a condition of conjunction when transition from the odd fold $z^{\prime}=1,3,5, \ldots$ to even fold $z^{\prime \prime}=0,2,4, \ldots$

Corresponding equations take the form as follows:

$\frac{m c^{2}}{\hbar \sqrt{1-\left(\frac{c_{z F}^{\prime \prime}}{c}\right)^{2}}}-\frac{\pi}{\Delta t_{0}} 2 z \sqrt{1-\frac{c_{z F}^{\prime \prime 2}}{c^{2}}}=2 \pi F^{\prime}\left(c_{z F}^{\prime \prime}\right)-\frac{m c^{2}}{\hbar \sqrt{1-\left(\frac{c_{z F}^{\prime \prime}}{c}\right)^{2}}}+\frac{\pi}{\Delta t_{0}}(2 z+1) \sqrt{1-\frac{c_{z F}^{\prime \prime 2}}{c^{2}}}$, $z=0,1,2,3, \ldots$

$\frac{m c^{2}}{\hbar \sqrt{1-\left(\frac{c_{z F}^{\prime}}{c}\right)^{2}}}-\frac{\pi}{\Delta t_{0}} 2 z \sqrt{1-\frac{c_{z F}^{\prime 2}}{c^{2}}}=2 \pi F^{\prime}\left(c_{z F}^{\prime}\right)-\frac{m c^{2}}{\hbar \sqrt{1-\left(\frac{c_{z F}^{\prime}}{c}\right)^{2}}}+\frac{\pi}{\Delta t_{0}}(2 z-1) \sqrt{1-\frac{c_{z F}^{\prime 2}}{c^{2}}}$, $z=1,2,3,4 \ldots$

In view of (10) and (12) we have $2 \pi F\left(c_{z F}\right)=\frac{\pi}{\Delta t_{0}} \sqrt{1-\left(\frac{c_{z F}}{c}\right)^{2}}$.

With due consideration of this equality the initial equations are reduced to the form as follows:

$\left\{\begin{array}{l}\frac{2 m c^{2}}{\hbar \sqrt{1-\left(\frac{c_{z F}^{\prime \prime}}{c}\right)^{2}}-(4 z+2) \frac{\pi}{\Delta t_{0}} \sqrt{1-\frac{c_{z F}^{\prime \prime 2}}{c^{2}}}=0, z=0,1,2,3, \ldots,} \\ \frac{2 m c^{2}}{\hbar \sqrt{1-\left(\frac{c_{z F}^{\prime \prime}}{c}\right)^{2}}}-4 z \frac{\pi}{\Delta t_{0}} \sqrt{1-\frac{c_{z F}^{\prime \prime 2}}{c^{2}}}=0, \quad z=1,2,3,4 \ldots .\end{array}\right.$

Having substituted here $\Delta t_{0}=\chi \pi t_{P l}=\frac{\pi \chi \hbar}{c^{2} m_{P l}}$ from (25), we shall obtain:

$$
\begin{aligned}
& \frac{c_{z F}^{\prime \prime}}{c}=\sqrt{1-\frac{\chi}{2 z+1} \frac{m}{m_{P l}}}, \quad z=0,1,2, \ldots, \\
& \frac{c_{z F}^{\prime}}{c}=\sqrt{1-\frac{\chi}{2 z} \frac{m}{m_{p l}}}, \quad z=1,2,3, \ldots .
\end{aligned}
$$


Let us determine the values of frequency (energy) at the conjunction points of the folds:

$$
\begin{aligned}
& \omega^{\prime \prime}\left(c_{z F}^{\prime \prime}\right)=\omega^{\prime}\left(c_{z F}^{\prime \prime}\right)=\frac{m c^{2}}{\hbar \sqrt{\frac{\chi}{2 z+1} \frac{m}{m_{P l}}}}-\frac{\pi}{\Delta t_{0}} 2 z \sqrt{\frac{\chi}{2 z+1} \frac{m}{m_{P l}}}= \\
& =\frac{c^{2}}{\hbar} \sqrt{\frac{m m_{P l}}{\chi(2 z+1)}}, \quad z=0,1,2, \ldots, \\
& \omega^{\prime}\left(c_{z F}^{\prime}\right)=\omega^{\prime \prime}\left(c_{z F}^{\prime}\right)=\frac{m c^{2}}{\hbar \sqrt{\frac{\chi}{2 z} \frac{m}{m_{P l}}}}-\frac{\pi}{\Delta t_{0}} 2 z \sqrt{\frac{\chi}{2 z} \frac{m}{m_{P l}}}=0, \quad z=1,2,3, \ldots .
\end{aligned}
$$

The frequency (energy) of a particle becomes zero in every point where the odd fold transfers into the even one. The largest value of particle energy with $m$ is at the conjunction point of zero and first folds that is with $\mathrm{z}=0$.

$$
E_{\text {sup }}=c^{2} \sqrt{\frac{m m_{P l}}{\chi}} .
$$

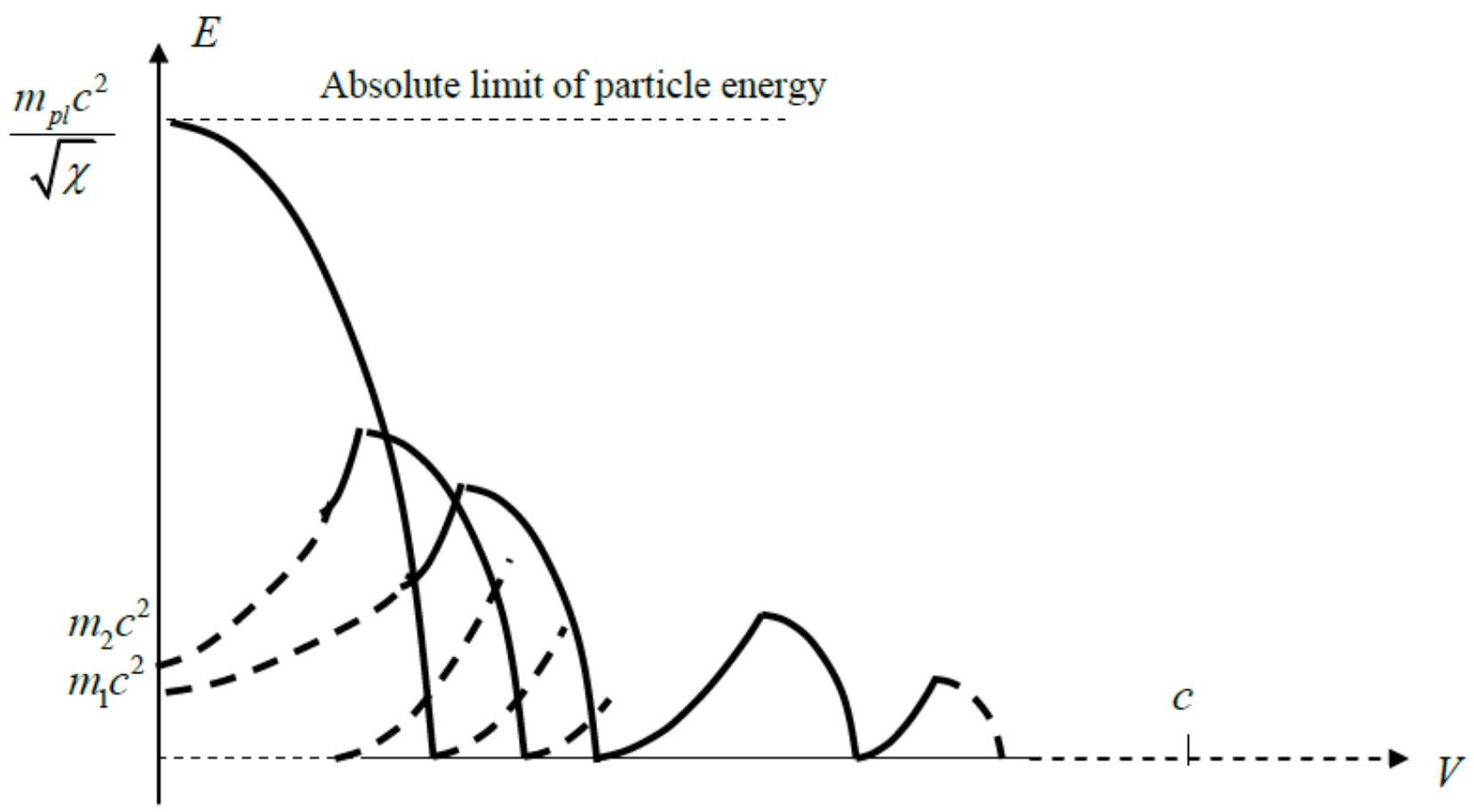

Fig. 3. Energy including wave function aliasing for the particles with different mass 
The value of dimensionless physical constant $\chi$ may be obtained immediately from the formula (33) upon instrumental detection of aliasing. Let us make preliminary estimation of this value. The observations of the ultra-high energy cosmic rays give reason to suppose that the order $10^{20} \mathrm{eV}$ is inherent in the ultimate proton energy. Having inserted $E_{\text {sup }}=10^{20} \mathrm{eV}$, the mass of proton $938 \mathrm{MeV}$ and Planckian mass $1,22 \cdot 10^{19} \mathrm{GeV}$ in (33) we shall obtain:

$$
\chi=\frac{m m_{P l} c^{4}}{E_{\text {sup }}^{2}}=\frac{\Delta t_{0}}{\pi t_{P l}} \sim 1,144 \cdot 10^{-3}
$$

This fundamental in its physics dimensionless constant is of the same order as a reduced fine structure constant $\frac{\alpha}{2 \pi}=1,161409732 \cdot 10^{-3}$ examination (the differe estimation of an order of magnitude, it may be assumed that in reality $\chi=\frac{\alpha}{2 \pi}$ and the ultimateparticle energy with the mass of the proton is equal to $0,985 \cdot 10^{20} \mathrm{eV}$

At the same time, three physical constants: time quantum $\Delta \mathrm{t}_{0}$, fine structure constant $\alpha$ and Planckian time $t_{P l}$ prove to be interconnected by a simple ratio:

$$
\Delta t_{0}=\alpha \frac{t_{P l}}{2}=7,297352 \cdot 10^{-3} \frac{5,39056 \cdot 10^{-44}}{2}=19,668407 \cdot 10^{-47} \mathrm{~s} .
$$

In other words, a fine structure constant presents double-time quantum-to-Planckian time ratio. Under the Markov [6] hypothesis the maximum possible mass of a particle at rest is equal to the Planckian mass. However, as it follows from (29), a theoretical mass limit may be set to $\frac{m_{P l}}{\chi}$ With this particle mass the frequency is substituted almost at its zero speed, the dynamics of a particle is abnormal at all speeds of its movement. Therefore, with a discrete time, no matter how close is the speed of movement of any particle to the speed of light, the quantity of its energy is limited to $E \leq \frac{E_{P l}}{\sqrt{\chi}}$ due to the phenomenon of frequency substitution.

The time may be considered continuous in the frequency (energy) domain where the aliasing doesn't come out. However, the nature of particle movement beyond the confines of this domain wherein its energy reduces with increase of the speed becomes paradoxical from the point of view of the hypothesis of continuous time.

With discrete time $(\chi \neq 0)$ the particle energy of discrete mass doesn't monotonically increase to infinity with increase of the speed of its movement due to substitution of its frequency. The energy line shown in the diagram of frequency (energy) dependence on speed is folded. And at the same time, the particle energy proves to be equal to zero at the bottom conjunction points. In view of the positive value of rest energy of unbound charged particle with mass $m$, we shall obtain that if it is accelerated up to speed $c_{1 F}^{\prime}$ for example, in the accelerating device, energy $E=m c^{2}$ is released. 
The particle is giving this energy to accelerating electromagnetic field. This technique of direct particle mass conversion into the electromagnetic field energy fundamentally differs from the techniques based on the reaction of fission, synthesis or annihilation of elementary particles. Generally speaking, the aliasing phenomenon implies a possibility in principle for the existence of massive particles with a disproportionately low kinetic energy. If the wave function aliasing is ignored, a false inference as regards the mass of these particles may be drawn as judged by the energy measurements. This leads to an illusory contradiction emerging as a result of observation where the mass of a particle comes out as the inertial and gravitational one.

For example, a massive ultra relativistic particle moving at a speed $c_{1 F}^{\prime}=c \sqrt{1-\frac{1}{4} \chi \frac{m}{m_{P l}}}$ in the magnetic field with a component of strength perpendicular to the direction of its movement does not radiate because the intensity of synchrotron radiation is proportional to

the square of a particle energy being equal to zero in the present case. Not manifesting itself by the electromagnetic wave radiation this particle nevertheless is interacting with the medium surrounding it through the gravitational field possibly being the essence of the dark matter problem. Due to aliasing a particle whose energy initially exceeds the Greisen-Zatsepin-Kuzmin threshold (GZK limit) to a considerable extent may move in space over all distances non-reacting with the microwave background radiation. This will take place if its speed exceeds the critical one (wherein the frequency is being substituted) to the extent that a particle energy will reduce lower than the GZK threshold limit being estimated by value $5 \cdot 10^{19} \mathrm{eV}$. If a particle movement subsequently slows prior to its detection, the energy will gain anew. This may lead to the changes in spectrum of highenergy particles referred to as a paradox of the GZK threshold limit.

\section{Conclusions}

1. In continuous time, the frequency (energy) of a particle grows unlimitedly when its speed approaches the speed of light in vacuum. In discrete time, on the contrary, due to aliasing, the frequency (energy) of a particle is limited and decreases when the speed grows above some crucial value and the first substitution of frequency takes place. If this phenomenon were found in an experiment, it would corroborate the hypothesis of time discreteness empirically.

2. Time discreteness causes an anomalous dynamics of particles, which has not been investigated before.

3. If time is discrete, then, in ultrarelativistic field, such particles speeds, different from zero, should exist, by which the frequency (energy) of wave function turns into zero.

4. The fact of energy decreasing under the value of immobility energy due to aliasing by its momentum, shows that there exists a possibility of creating some principally new energy sources; it can also explain some cosmological paradoxes, such as, for example, the ones of GZK paradox and dark matter.

\section{References}

[1] Esposito S., Salesi G., arXiv:1001.0050v1 [physics. hist-ph], 2009

[2] Giovanni Amelino-Camelia, arXiv:gr-qc/0012051v2, 2000

[3] Guang Ping He, arXiv:0911.2416v2 [quant-ph], 2009

[4] Shan Gao, arXiv: 1001.5085v2 [physics.gen-ph], 2011.

[5] R. Otnes, L. Enochson, 1978. Applied time series analysis. Basic techniques. - John Wiley and Sons, Inc: 428.

[6] Markov M.A. // Progr. Theor. Phys.: Suppl. Commemoration Issue for 30th Anniversary of the Meson Theory by Dr. H. Yukawa, 1965. 